\title{
STRONGLY GENERALIZED RADICAL SUPPLEMENTED MODULES
}

\author{
SOUMitra Das \\ AND \\ Ardeline M. Buhphang \\ Department of Mathematics \\ North-Eastern Hill University \\ Shillong-793022, Meghalaya, India \\ e-mail: soumitrad330@gmail.com \\ ardeline17@gmail.com
}

\begin{abstract}
We introduce and study strongly generalized radical-supplemented modules (or briefly sgrs-modules). With the notation $\operatorname{Rad}_{g}(R):=\cap\{K: K \leq$ $R_{R}, K$ is both essential and maximal\}, we prove that (under some mild conditions on a ring $R$ ) every right $R$-module is a sgrs-module if and only if $\frac{R}{\operatorname{Soc}(R)}$ is right perfect and the idempotents lift module $\operatorname{Rad}_{g}(R)$.
\end{abstract}

Keywords: essential submodules, supplemented modules, strongly radicalsupplemented modules, (semi-) perfect rings.

2010 Mathematics Subject Classification: Primary 16D10; Secondary 16D40, 16D70, 16D60.

\section{REFERENCES}

[1] F.W. Anderson and K.R. Fuller, Rings and Categories of Modules (New York, Springer-Verlag, 1974).

[2] E. Büyükaşik and E. Türkmen, Strongly radical supplemented modules, Ukrainian Math. J. 63 (2011) 1306-1313.

doi:10.1007/s11253-012-0579-3

[3] J. Clark, C. Lomp, N. Vanaja and R. Wisbauer, Lifting Modules: Supplements and Projectivity in Module Theory (Basel, Boston, Berlin, Birkhauser Verlag, 2006). doi:10.1007/3-7643-7573-6 
[4] B. Koşar, C. Nebiyev and A. Pekin, A generalization of g-supplemented modules, Miskolc Math. Notes 20 (2019) 345-352.

doi:10.18514/MMN.2019.2586

[5] B. Koşar, C. Nebiyev and N. Sökmez, G-supplemented modules, Ukrainian Math. J. 67 (2015) 861-864.

doi:10.1007/s11253-015-1127-8

[6] M.T. Koşan, $\delta$-lifting and $\delta$-supplemented modules, Algebra Colloq. 14 (2007) 53-60.

doi:10.1142/S1005386707000065

[7] C. Lomp, On semilocal modules and rings, Comm. Algebra 27 (1999) 1921-1935. doi:10.1080/00927879908826539

[8] C. Nebiyev and H. Hüseyin Ökten, Weakly G-supplemented modules, European J. Pure Appl. Math. 10 (2017) 521-528.

https://ejpam.com/index.php/ejpam/article/viewFile/2662/534

[9] T.C. Quynh and P.H. Tin, Some properties of e-supplemented and e-lifting modules, Vietnam J. Math. 41 (2013) 303-312.

doi:10.1007/s10013-013-0022-6

[10] L.V. Thuyet and P.H. Tin, Some characterizations of modules via essentially small sub-modules, Kyungpook Math. J. 56 (2016) 1069-1083. doi:10.5666/kmj.2016.56.4.1069

[11] B.N. Türkmen and E. Türkmen, On a generalization of weakly supplemented modules, An. Ştiinţ. Univ. Al. I. Cuza Iaşi. Mat. (N.S.) 63 (2017) 441-448.

doi:10.1515/aicu-2015-0012

[12] R. Wisbauer, Foundations of Module and Ring Theory (Gordon and Breach, Philadelphia, 1991).

[13] Y. Zhou, Generalizations of perfect, semiperfect, and semiregular rings, Algebra Colloq. 7 (2000) 305-318. doi:10.1007/s10011-000-0305-9

[14] D.X. Zhou and X.R. Zhang, Small-essential submodules and morita duality, Southeast Asian Bull. Math. 35 (2011) 1051-1062. http://www.seams-bull-math.ynu.edu.cn/archive.jsp

[15] H. Zöschinger, Komplementierte moduln über dedekindringen, J. Algebra 29 (1974) $42-56$. doi:10.1016/0021-8693(74)90109-4

Received 16 May 2019

Revised 31 October 2019

Accepted 27 January 2020 